\title{
La relation structure-activité d'analogues de rhynchosporosides. I - Synthèse chimique.
}

\author{
Delphin RAFENOMANANJARA, Pierre AURIOL (*) \& Christian MAZARS (*) \\ Station Marine, BP 141, Tulear, Madagascar, \\ (*) Université Paul Sabatier, Centre de Physiologie végétale, Laboratoire associé au C.N.R.S. $n^{\circ}$ 24I, I18, \\ route de Narbonne, F 31062 Toulouse Cedex.
}

RÉSUMÉ

\begin{abstract}
Rhynchosporium secalis, agent pathogène responsable de la rhynchosporiose de l'orge, produit une famille de composés phytotoxiques : les rhynchosporosides, $\alpha-0-1$ glucosides de propanediol. La synthèse chimique d'analogues structuraux du glucoside et du cellobioside a été réalisée. Ils ne diffèrent des composés naturels que par le mode d'attache de la partie glucidique au propanediol ou par l'utilisation d'un autre aglycone (isopropanol, n-propanol, n-butanol).
\end{abstract}

Mots-clés additionnels : Rhynchospinum secalis, Pureté, Structure.

Rhynchosporium secalis, the causal agent of barley leaf scald, produces a family of phytotoxins : the rhynchosporosides which are $\alpha-0-1$ glucosides of propanediol. Chemical synthesis of structural analogs of the glucoside and cellobioside was achieved. They differ from the natural toxins by the propanediol connection point or by the utilization of an other aglycone moiety (isopropanol, n-propanol, n-butanol).

Additional key-words : Rhynchosporium secalis, Purity, Structure.

\section{INTRODUCTION}

Rhynchosporium secalis (Oud.) Davis est l'agent pathogène responsable de la rhynchosporiose de l'orge. Ce parasite produit une phytotoxine dont la structure initialement proposée a été celle d'un $\alpha-0-2$ cellobioside de propanediol (AURIOL et al., 1978). Des études ultérieures (BEIER, 1979; STROBEL, 1982) ont montré que ce microorganisme synthétisait en réalité un mélange de $1-0-\alpha-D$ glucopyranoside, 1-0- $\alpha-D$ cellobioside, $1-0-\alpha-D$ cellotrioside de propanediol et que la molécule initialement isolée correspondait en fait au cellotrioside. Nous avons, au laboratoire, confirmé (D. ANDURAND comm. pers., 1981) l'existence de cette famille et démontré (MAZARS, 1982) qu'elle pouvait s'étendre à des termes de polymérisation supérieure à trois glucoses.

Dans le but ultérieur de définir des caractéristiques structurales nécessaires ou favorables à l'expression de l'activité de ces molécules nous avons, dans une première étape, tenté la synthèse d'analogues de certaines d'entre elles. Nous présentons ici des résultats relatifs à la synthèse chimique d'analogues du glucoside et du cellobioside naturels, ces deux der-

(*) Auteurs auxquels doit être adressée la correspondance relative à cet article. niers s'avérant capables (BEIER, 1979 ; MAZARS, 1982) d'induire chez la plante hôte certains des symptômes caractéristiques de la maladie.

\section{MÉTHODES EXPÉRIMENTALES}

\section{A. Obtention des aglycones *}

Les aglycones suivants ont été utilisés : n-propanol, isopropanol, n-butanol (Merck) et le monolaurate de propylène obtenu par purification par chromatographie sur colonne d'acide silicique d'un mélange commercial (Fluka).

\section{B. Synthèse des osides}

A la fois pour les glucosides et pour les cellobiosides nous avons utilisé la séquence de réactions suivante :

\section{Péracétylation}

Elle a été effectuée selon le protocole expérimental proposé par FRITZ \& SCHENK (1959).

\footnotetext{
* Quand l'origine commerciale n'est pas mentionnée les solvants et produits sont fournis par Prolabo.
} 


\section{Bromation}

Nous avons retenu le procédé décrit par BARZAï-MARTOS \& KÖRÖSY (1950) que nous avons légèrement modifié en remplaçant, comme solvant de réaction, l'anhydride acétique et l'acide acétique glacial par le chloroforme.

\section{Condensation}

Le bromacétoglucose ou le bromoacétocellobiose sont condensés avec les différents aglycones dans un milieu réactionnel à base de chloroforme absolu ; la condensation est catalysée par l'iode en présence d'oxyde d'argent selon la réaction de Koenigs-Knorr adaptée par RAFENOMANANJARA (1979).

\section{Anomérisation}

C'est la méthode préconisée par PACSU et al., (1963) utilisant le tétrachlorure de titane que nous avons retenue.

\section{Desacétylation}

Elle a été opérée par le méthoxyde de sodium dans le méthanol comme le décrivent THOMPSON et al., (1963).

Remarque: Après anomérisation et désacétylation, la pureté des composés obtenus a été vérifiée par chromatographie en couche mince de silicagel (Merck) avec les systèmes solvants : benzène - acétate d'éthyle $(8-2 ; \mathrm{v} / \mathrm{v})$ suivie d'une migration dans : benzène-acétate d'éthyle $(9-1 ; \mathrm{v} / \mathrm{v})$ pour les glucosides et benzène-méthanol $(95-5 ; \mathrm{v} / \mathrm{v})$ suivie de 2 migrations dans : benzène-acétate d'éthyle $(9-1 ; v / v)$ pour les cellobiosides. La révélation a été opérée en utilisant l'acide sulfurique à $10 \mathrm{p} .100$ dans l'éthanol $(10 \mathrm{mn}$, $100^{\circ} \mathrm{C}$ ).

\section{Hydrolyses}

\section{Hydrolyse totale}

$5 \mathrm{mg}$ d'analogue synthétisé sont mis dans un tube en présence de $0,5 \mathrm{ml}$ d' $\mathrm{HCl} 2 \mathrm{~N}$; le tube scellé est alors porté $1 \mathrm{~h}$ au bain marie bouillant. Après refroidissement et ouverture du tube, on ajoute une goutte d'éther éthylique qui servira de standard interne pour la chromatographie en phase gazeuse.

L'identification des alcools libérés par l'hydrolyse est alors effectuée par chromatographie en phase gazeuze (Perkin. Elmer 900); le volume d'injection est de $0,7 \mu \mathrm{l}$ d'hydrolysat ; la colonne capillaire $(1800 \times 1 \mathrm{~mm})$ contient du carbowax $20 \mathrm{M}$; la température de la colonne est de $85^{\circ} \mathrm{C}$ et celle de l'injecteur de $320^{\circ} \mathrm{C}$; la détection se fait par ionisation de flamme et le gaz vecteur est l'hélium.

\section{Hydrolyse ménagée}

On part de $5 \mathrm{mg}$ de composé auxquels on ajoute $1 \mathrm{ml}$ d' $\mathrm{HCl} \mathbf{N}$ dans un tube qui est alors scellé et porté au bainmarie bouillant pendant $20 \mathrm{mn}$. Ensuite l'hydrolysat est passé à travers une colonne contenant $1,5 \mathrm{~cm}^{3}$ de résine Dowex 2, forme $\mathrm{CH}_{3} \mathrm{COO}^{-}$. La solution ainsi neutralisée est évaporée à sec 2 fois successivement avec addition d'eau distillée ; après la $2^{\mathrm{e}}$ évaporation, le résidu sec est repris par $0,5 \mathrm{ml}$ d'eau. $4 \mu \mathrm{l}$ de cette solution sont alors déposés sur une couche mince de gel de silice (Merck) et chromatographiés dans le système solvant : n-propanol-acétate d'éthyle $\mathrm{H}_{2} \mathrm{O}$-pyridine (7-6-1-1; v/v).

\section{Hydrolyse enzymatique}

Les analogues $(50 \mu \mathrm{g})$ du cellobioside naturel sont incubés pendant $6 \mathrm{~h}, \grave{a} 37^{\circ} \mathrm{C}$, dans $100 \mu \mathrm{l}$ de tampon acétate $50 \mathrm{mM}, \mathrm{pH} 5,0$ en présence de $50 \mu \mathrm{g}$ de $\beta$-glucosidase (Almond $\beta$-glucosidase, E.C. 3.2.1. 21, Sigma). La réaction est arrêtée par addition de 0,1 g de Dowex 50, 200-400 mesh, forme $\mathrm{H}^{+}$. Après filtration sur papier, la solution est chromatographiée dans des conditions identiques à celles utilisées après l'hydrolyse ménagée.

\section{III.RÉSULTATS}

\section{A. Contrôle de pureté du monolaurate de propylène}

L'élution par des passages successifs de chloroforme et de mélanges chloroforme-éther éthylique de la colonne d'acide silicique a permis d'isoler 5 ensembles de fractions dont l'un (éluat IV) ne contenant qu'un seul composé. Nous avons alors tenté de déterminer la nature de cette molécule.

Par spectrophotométrie infrarouge (Beckman Ir 20), il apparaît que cet éluat (fig. 1) présente une absorption à $3400 \mathrm{~cm}^{-1}$ caractéristique de la fraction hydroxyle, un double pic, entre 2900 et $3000 \mathrm{~cm}^{-1}$, indiquant la présence de groupement méthyle et méthylène, enfin un pic à $1740 \mathrm{~cm}^{-1}$ démontrant la présence dans l'échantillon de la fonction ester carboxylique.



Figure 1

Spectre d'absorption infra-rouge de l'éluat IV. Le spectre a été réalisé en utilisant, entre lames de $\mathrm{CaF}_{2}$, l'échantillon en solution dans l'eau. Infrared spectrum of the eluted fraction IV. The spectrum has been performed on a sample water solution between $\mathrm{CaF}_{2}$ blades.

Par résonance magnétique nucléaire (Hitachi E 360), nous avons obtenu un spectre (fig. 2) qui donne un signal complexe ( $\delta: 0,95$ p.p.m ; intégrant pour 3 protons) correspondant au méthyle terminal de l'acide laurique ; un doublet ( $\delta: 1,20 ; 3$ protons) correspondant au méthyle du propanediol ; un signal complexe ( $\delta: 1,30 ; 18$ protons) correspondant à la chaîne aliphatique de l'acide laurique ; un signal complexe $(\delta: 2,30 ; 2$ protons) correspondant au $\mathrm{CH}_{2}$ de l'acide laurique vicinal du carbone d'attache au propanediol ; un signal complexe ( $\delta: 3,90 ; 3$ protons) correspondant aux carbones 1 et 2 du propanediol.

L'ensemble de ces informations confirme que la molécule présente dans l'éluat IV est bien du laurate de (hydroxy-2) propyle ou monolaurate de propylène. 


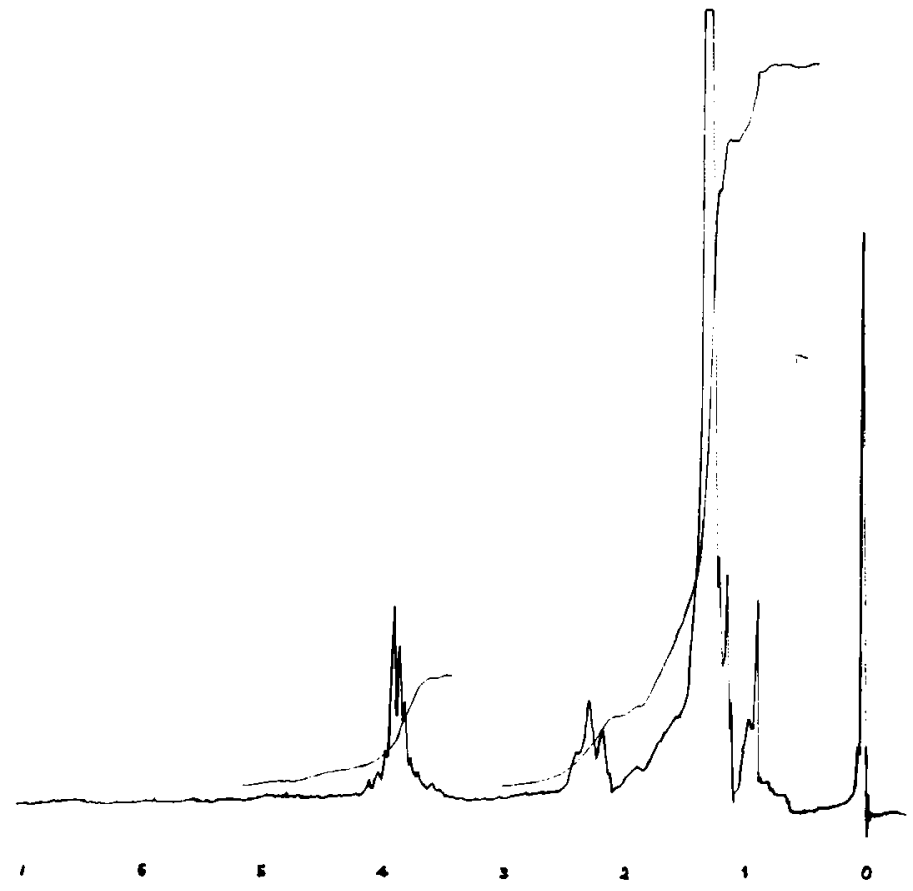

Figure 2

Spectre de résonance magnétique nucléaire du monolaurate à $60 \mathrm{MHz}$; le solvant est $\mathrm{CCl}_{4}$; le standard interne est le T.M.S.

$60 \mathrm{MHz}$ proton magnetic resonance spectrum of the monolaurate; the solvent is $\mathrm{CCl}_{4}$; internal standard is T.M.S.



Figure 3

Chromatographie en phase gazeuse d'hydrolysats totaux de $\beta$-cellobiosides et d'a-glucosides (cf. légende dans le texte). Les témoins sont respectivement le $n$-butanol $(B u)$; le n-propanol $\left(P_{l}\right)$; l'isopropanol $\left(P_{2}\right)$ et l'éther $(E)$ utilisé comme standard interne.

Gas chromatography of $\beta$-cellobiosides and $\alpha$-glucosides after total hydrolysis (see text for legend). The controls are respectively $n$ butanol (Bu); n-propanol $\left(P_{1}\right)$; isopropanol $\left(P_{2}\right)$ and ether $(E)$ utilised as internal standard.

\section{B. Contrôle de l'identité des diverses molécules synthétisées}

Afin de confirmer la nature des produits obtenus, nous les avons soumis à des hydrolyses totales pour déceler la présence des alcools aglycones et à des hydrolyses ménagées pour vérifier la composition de la partie osidique. Enfin, l'obtention des anomères a été prouvée par résonance magnétique nucléaire et par hydrolyse enzymatique.

\section{Hydrolyses totales}

Des résultats obtenus (fig. 3 ) on peut tirer les conclusions suivantes : il existe un très bonne correspondance entre les temps de rétention des différents alcools issus des hydrolysats et ceux servant de référence. Ces expériences confirment donc la présence de propanol, isopropanol et butanol dans les composés de synthèse. En ce qui concerne le propanediol, dans les conditions chromatographiques indiquées, il n'a pas pu être identifié ; nous avons alors recherché et démontré sa présence dans les molécules de synthèse (issues du monolaurate de propylène comme précurseur d'aglycone) par chromatographie en couche mince sur plaque de gel de silice (Merck) dans le mélange solvant: benzène-éther éthylique-méthanol $(8-1,5-0,5 ; \mathrm{v} / \mathrm{v})$.

\section{Hydrolyses ménagées}

Les résultats de ces hydrolyses (fig. 4) montrent que la dissection chimique a libéré des composés qui s'accordent avec les structures proposées; en effet, pour les glucosides apparaissent du glucose et du glucoside non hydrolysé ; pour les cellobiosides on peut observer du glucose, du cellobiose, le glucoside correspondant et le cellobioside restant non hydrolysé ; seul l'alcool constituant l'aglycone n'est pas

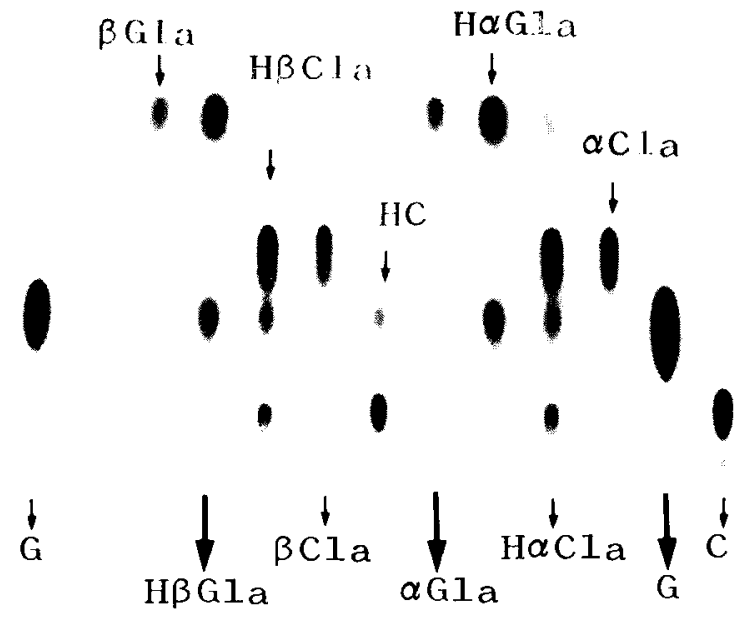

Figure 4

Chromatographie sur plaque de gel de silice d'hydrolysats ménagés $(H)$ $d^{\prime} \alpha$ et de $\beta$-glucosides et cellobiosides de propanediol (cf. légende dans le texte) et de témoins ( $G:$ glucose ; $C$ : cellobiose).

Chromatography on silica gel plate of mild hydrolysis products $(H)$ of $\alpha$ and $\beta$ propanediol glucosides and cellobiosides (see text for legend) and controls $(G:$ glucose; $C$ : cellobiose).

détecté du fait de sa faible concentration. Pour les autres glucosides et cellobiosides de synthèse des résultats identiques, après hydrolyse ménagée, ont été obtenus. Dans tous les cas, la pureté des composés testés est apparue, comme dans la figure 4 , nettement. 


\section{Vérification de l'obtention des anomeres}

Pour vérifier l'efficacité de la méthode d'anomérisation que nous avons retenue (PACSU et al., 1963) nous avons réalisé le spectre RMN de 2 glucosides pris comme exemples : $\alpha$ et $\beta$-glucoside d'isopropyle ; par ailleurs, les cellobiosides obtenus ont été soumis à l'hydrolyse enzymatique par la $\beta$-glucosidase.

Le spectre RMN du $\beta$-glucoside (fig. 5) donne 2 doublets très proches $(\delta: 1,15$ et : 1,25 p.p.m; intégrant pour 6 protons) correspondant aux 2 méthyles de l'isopropanol); des signaux complexes ( $\delta: 3,10$ à 4,$35 ; 7$ protons) correspondant aux hydrogènes portés par les carbones 2 à 6 du glucose et par le carbone 2 de l'isopropyle; enfin un doublet $(\delta: 4,50 ; \mathrm{J}: 7,5 \mathrm{~Hz} ; 1$ proton) confirmant la présence d'un proton anomérique $\beta$, en accord avec VAN DER VEEN (1962).



5

4

2

Figure 5

Spectre de résonance magnétique nucléaire de $\beta-G P_{2}$ à $60 \mathrm{MHz}$; le solvant est l'eau deutériée; le spectre est centré à 4,75 ppm par rapport au pic donné par $\mathrm{H}_{1}^{1} \mathrm{H}_{2}^{2} \mathrm{O}$.

A $60 \mathrm{MHz}$ proton magnetic resonance spectrum of $\beta-G P_{2}$ in deuteried water; the spectrum is referenced internally to $H_{I}^{1} H_{2}^{2} O$ at $\delta 4.75$.

Le spectre RMN du composé anomérisé (fig. 6) proposé comme $\alpha$-glucoside d'isopropyle présente les mêmes caractéristiques spectrales à l'exception de l'absence d'un doublet à 4,5 p.p.m et de la présence d'un doublet ( $\delta: 5,0$; $\mathrm{J}: 3 \mathrm{~Hz} ; 1$ proton), en accord avec la présence d'un proton $\alpha$-anomérique selon VAN DER VEEN (1962).

Les résultats obtenus après hydrolyse enzymatique sont commentés dans la discussion.

En résumé, nous avons obtenu 8 ( $\alpha$ et $\beta$ ) analogues du rhynchosporoside 1 : G-La, à aglycone propanediol lié en 2 (issu de la condensation glucose-propylène 1-monolaurate) ;

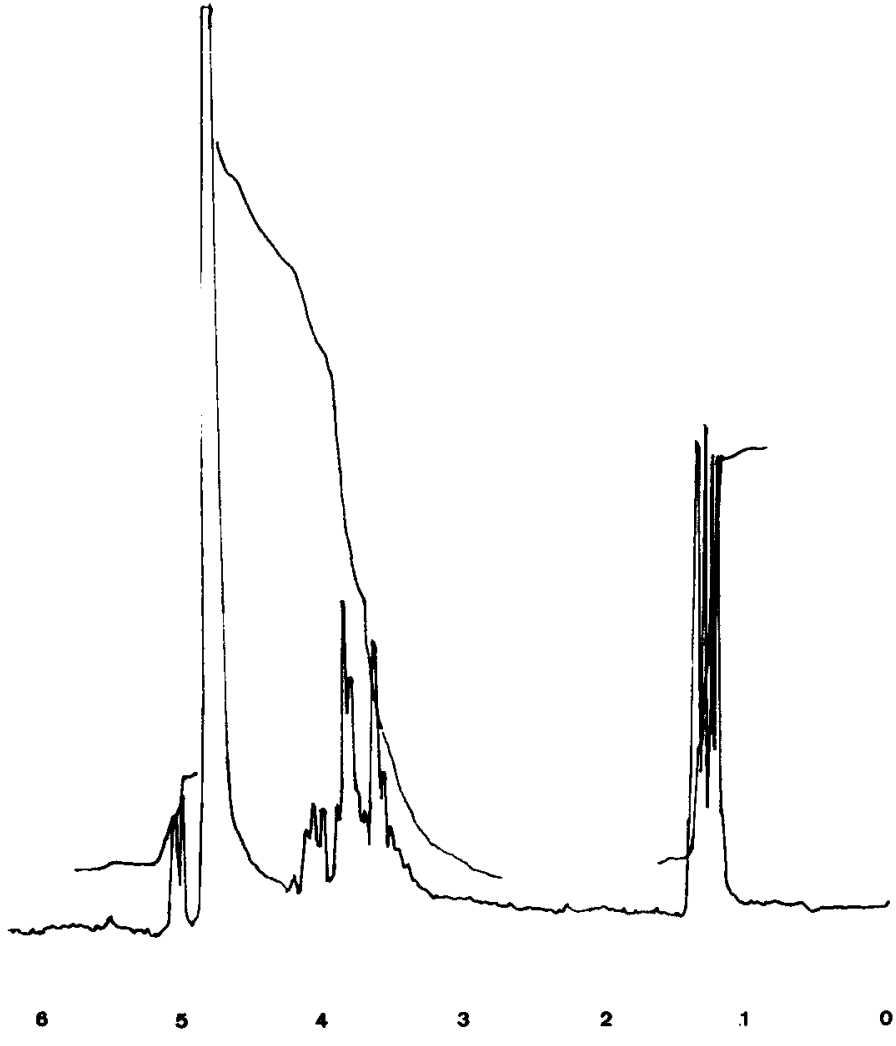

Figure 6

Spectre de résonance magnétique nucleaire de l' $\alpha-G P_{2}$ a $60 \mathrm{MHz}$; le solvant est l'eau deutérié; le spectre est centré à 4,75 ppm par rapport au pic donné par $\mathrm{H}_{1}^{1} \mathrm{H}_{2}^{2} \mathrm{O}$.

A $60 \mathrm{MHz}$ proton magnetic resonance spectrum of $\alpha$-GP $\mathrm{P}_{2}$ in deuteried water; the spectrum is referenced internally to $\mathrm{H}_{I}^{I} \mathrm{H}_{2}^{2} \mathrm{O}$ at $\delta 4.75$.

G- $P_{2}$, aglycone isopropanol lié en 2 ; G-P $\mathrm{P}_{1}$, aglycone $\mathrm{n}$ propanol lié en 1 ; G-Bu : aglycone n-butanol lié en 1 et les 8 cellobiosides correspondants: C-La, C-P, C-P, $\mathrm{C}-\mathrm{Bu}$.

\section{DISCUSSION ET CONCLUSION}

L'objectif de ce travail étant l'obtention d'analogues structuraux de rhynchosporosides, nous avons été conduits à utiliser, dans ce but, des méthodes classiques de synthèse organiques, en particulier au cours d'étapes de glycosidation et d'anomérisation.

Au cours de la réaction d'anomérisation des celliobiosides, au niveau de liaison avec l'aglycone, il peut se produire une anomérisation potentielle de la liaison glucosidique $\beta-1$ $\rightarrow 4$ établie entre les 2 glucoses de la molécule de cellobiose. Au terme de la réaction d'anomérisation, nous avons obtenu 4 précipités successifs par maintien à $0^{\circ}-4^{\circ} \mathrm{C}$ de la solution éthanolique finale ; chaque précipité, après désacétylation, a été soumis à hydrolyse enzymatique par la $\beta$-glucosidase, dans les conditions décrites. Seul un de ces précipités a été totalement hydrolysé en glucose et glucoside ce qui signifiait que sa partie glucidique était totalement constituée de cellobiose et qu'il était exempt du maltoside correspondant, le rendement en $\alpha$-cellobioside pur étant environ de 25 p. 100. 
L'efficacité de l'anomérisation de la liaison cellobiose aglycone a pu être démontrée par chromatographie comparée ; en effet, à l'issue des synthèses des cellobiosides péracétylés, leur comportement chromatographique, avant et après anomérisation, était différent. Dans la mesure où ces composés une fois désacétylés montraient une composition identique aux $\beta$-cellobiosides (cf. fig. 4), il était fort probable qu'il s'agissait de $\alpha$-cellobiosides correspondants.

Quant à l'efficacité de l'anomérisation des $\beta$-glucosides, les résultats obtenus par analyse de résonance magnétique nucléaire l'ont clairement démontrée.

Enfin, tous les analogues que nous avons synthétisés se sont révélés chromatographiquement purs, sans traces de contamination apparente.
L'étude de la relation entre la structure de ces molécules, analogues chimiques de rhynchosporosides, et leur activité toxique constitue la $2^{e}$ étape de ce travail.

Reçu le 14 septembre 1982. Accepté le 2 décembre 1982.

\section{REMERCIEMENTS}

Les auteurs remercient vivement Mme E. COSTES qui a réalisé le spectre infra-rouge ainsi que les responsables du Département de Chimie de l'Université Paul Sabatier où ont été effectués les différents spectres de résonance magnétique nucléaire.

\section{RÉFÉRENCES BIBLIOGRAPHIQUES}

Auriol P., Strobel G., Pio Beltran J., Gray G., 1978. Rhynchosporoside, a host selective toxin produced by Rhynchosporium secalis, the causal agent of scald disease of barley. Proc. natl. Acad. Sci., USA, $75,4339-4343$.

Barczaï-Martos M., Körösy F., 1950. Preparation of acetobromesugars. Nature, 165, 369.

Beier R.C., 1979. Carbohydrate chemistry. Synthetic and structural investigations of the phytotoxins found in Helminthosporium sacchari and Rhynchosporium secalis. Ph. D. Thesis, Montana State University, Bozeman, Montana, 263 p.

Fritz J.S., Schenk G.H., 1979. Acid-catalized acetylation of organic hydroxyl groups. Anal. Chem., 31, 1808-1812.
Mazars C., 1982. Approche biochimique du rôle des rhynchosporosides dans l'interaction parasitaire. Rhynchosporium secalis-Hordeum vulgare. Thèse Spécialité, Physiol. vég., Toulouse, $71 \mathrm{p}$.

Pacsu E., Janson J., Linberg B., 1963. Anomerization. p. 376-385. In R.L. Whistler \& M.L. Wolfrom. Methods in carbohydrate chemistry. II. Acad. Press. N.Y. et Londres, 572 p.

Rafenomananjara D., 1979. Etude de proprietés biologiques d'une phytotoxine fongique (La rhynchosporoside) et d'analogues de synthese. Thèse spécialité Physiol. vég., Toulouse, 73 p.

Thompson A., Wolfrom M.L., Pacsu E., 1963. Deacetylation. p. 215-220. In R.L. Whistler \& M.L. Wolfrom. Methods in carbohydrate chemistry. II. Acad Press. N.Y. et Londres, $572 \mathrm{p}$.

Van der Veen J.M., 1962. An n.m.r. study of the glycosidic link in glycosides of glucose and galactose. J. organ. Chem., 28, 564-566. 